\title{
Identification of a Major Gene Responsible for Type 1 Diabetes in the Komeda Diabetes-Prone Rat
}

\author{
Norihide YOKOI \\ Division of Cellular and Molecular Medicine, Kobe University Graduate School of Medicine, \\ Chuo-ku, Kobe 650-0017, Japan
}

\begin{abstract}
Type 1 diabetes mellitus is an autoimmune disease involving both environmental and genetic factors. Genetic analyses in humans and rodents have shown that the major histocompatibility complex $(\mathrm{MHC})$ is a major genetic factor and that several other genes may be involved in the development of the disease. We performed genetic analysis of type 1 diabetes in a newly established animal model, the Komeda diabetes-prone (KDP) rat, and found that most of the genetic predisposition to diabetes is accounted for by two major susceptibility genes, MHC and Iddm/kdp1. In addition, we identified a nonsense mutation in the Casitas B-lineage lymphoma $b$ (Cblb) gene by positional cloning of $\mathrm{lddm} / \mathrm{kdp} 1$. In this paper, I review our positional cloning analysis of Iddm/kdp1 and propose a two-gene model of the development of type 1 diabetes in which two major susceptibility genes, Cblb and MHC, determine autoimmune reaction and tissue specificity to pancreatic $\beta$-cells, respectively.
\end{abstract}

Key words: autoimmune disease, Cblb gene, Komeda diabetes-prone rat, major histocompatibility complex, type 1 diabetes

Introduction
Most type 1 diabetes mellitus has autoimmune patho-
genesis by which insulin secreting pancreatic $\beta$-cells
are destroyed by the patient's own lymphocytes. While
both environmental and genetic factors are involved,
the major histocompatibility complex (MHC) has been
identified as the only major genetic factor, although
several other genes are thought to be minor factors [4].
Several spontaneous animal models of type 1 diabe-
tes have been developed and genetically analyzed. In
the non-obese diabetic (NOD) mouse, more than eigh-

teen loci have been reported, and the MHC was identified as the only major factor [14]. In the BioBreeding (BB) rat, at least six loci have been mapped, and two major loci, MHC and Lyp, are involved. A frameshift mutation in the immune-associated nucleotide 4 like 1 (Ian4l1) gene has been identified in Lyp [7, 12]. Although the genes of most other responsible loci have not yet been identified, these findings suggest that different combinations of genes may contribute to the development of diabetes in several animal models.

The Komeda diabetes-prone (KDP) rat is a newly established animal model in type 1 diabetes research

The author, Dr. Norihide YOKOI, was awarded the young investigator prize of JALAS in 2002.

(Received 27 September 2004 / Accepted 10 December 2004)

Address corresponding: N. Yokoi, Division of Cellular and Molecular Medicine, Kobe University Graduate School of Medicine, Chuo-ku, Kobe 650-0017, Japan 
[10]. We performed genetic analysis of type 1 diabetes in the KDP rat, and found that most of the genetic predisposition to diabetes could be accounted for by two major susceptibility genes, MHC and $I d d m / k d p I$ [15]. We also identified a nonsense mutation in the Casitas B-lineage lymphoma b (Cblb) gene by positional cloning of $I d d m / k d p l$ [16].

In this paper, I review our positional cloning analysis of $I d d m / k d p l$ and propose a two-gene model of the development of type 1 diabetes in the KDP rat.

\section{Komeda diabetes-prone (KDP) rats}

The KDP rat was established by Komeda and colleagues [10] as a diabetes-prone substrain of the Long-Evans Tokushima Lean (LETL) rat [8]. The phenotypic features of the KDP rat are autoimmune destruction of the pancreatic $\beta$-cells, rapid onset of overt diabetes with no sex difference, and lack of significant T-cell lymphopenia. Thus it resembles human type 1 diabetes. Most of the animals show moderate to severe lymphocyte infiltration into pancreatic islets (insulitis), and about $80 \%$ develop diabetes within 220 days of age [10]. However, the maintenance and production of KDP rats for use in research has been a continuing problem due to the poor reproductive ability of the animals. To solve this, we recently established the KDP rat as a segregating inbred strain having the $I d d m / k d p l$ region in the heterozygous state [17]. About $80 \%$ of $I d d m / k d p l$ homozygous rats develop diabetes with severe insulitis, while neither heterozygous nor wild-type rats develop diabetes.

\section{Genetic analysis of type 1 diabetes in the KDP rat}

\section{Inheritance of type 1 diabetes}

Genetic analysis of the LETL rat shows that a gene closely linked with the MHC is involved in insulitis [8], suggesting involvement of the MHC in the diabetes of the KDP rat. Accordingly, we used Tester Moriyama (TM) and Long-Evans Tokushima Otsuka (LETO) rats of the same MHC haplotype as KDP rats, and Brown Norway (BN) rats of a different haplotype to generate three backcrosses: $(\mathrm{TM} \times \mathrm{KDP}) \mathrm{F} 1 \times \mathrm{KDP}$, $(\mathrm{LETO} \times \mathrm{KDP}) \mathrm{F} 1 \times \mathrm{KDP}$, and $(\mathrm{BN} \times \mathrm{KDP}) \mathrm{F} 1 \times \mathrm{KDP}$ (TM cross, LETO cross, and BN cross) [15].
Diabetes was not observed in F1 but was observed in $\mathrm{N} 2$ rats. The cumulative frequency of diabetes in N2 rats varied with the cross: at 120 days of age, 32 and $34 \%$ of the animals in the TM and LETO crosses developed diabetes, respectively, while $16 \%$ of the animals in the $\mathrm{BN}$ cross developed diabetes. The cumulative frequency of diabetes in the TM and LETO crosses was about half that of KDP, while that of the BN cross was about one fourth, suggesting that the inheritance of diabetes might be explained by segregation of a single recessive gene in the TM and LETO crosses and by segregation of two such genes in the $\mathrm{BN}$ cross. If that was the case, then one of the two genes should be the MHC.

Genome wide scan for type 1 diabetes susceptibility genes

We first performed a genome wide scan using the TM cross, and successfully mapped one recessive gene, $I d d m / k d p 1$, on a $9 \mathrm{cM}$ genomic segment between Mox2 and D11M16Mit46 on rat chromosome 11 [15]. We genotyped $\mathrm{N} 2$ rats generated from LETO and BN crosses and found strong evidence of linkage to diabetes in the same region on chromosome 11 , confirming the recessive locus $I d d m / k d p 1$. In addition, we also found evidence of linkage to diabetes in the MHC region on chromosome 20 in the $\mathrm{BN}$ cross. These results demonstrate that $I d d m / k d p l$ is a major susceptibility gene for type 1 diabetes, and that most of the genetic predisposition to diabetes in these crosses is accounted for by the segregation of $I d d m / k d p l$ and MHC [15].

Comparative mapping of the Iddm/kdpl region in rat, mouse and human

We performed comparative mapping of the Iddm/ $k d p l$ region in rat, mouse and human, and demonstrated that orthologues of $I d d m / k d p l$ are located in the region between Gap43 and D16Mit46 on mouse chromosome 16 and in the p11-q13 region on human chromosome 3 [15]. As susceptibility genes for type 1 diabetes have not been mapped on these chromosomal regions in human and mouse, $I d d m / k d p l$ is believed to be a novel susceptibility gene for the disease.

\section{Positional cloning of Iddm/kdp1}

Fine genetic mapping of Iddm/kdpl

To clone $I d d m / k d p l$ by positional cloning strategy, 
we first constructed a precise genetic and comparative map of rat chromosome 11 and mouse chromosome 16 in the vicinity of the $I d d m / k d p l$ locus [16]. Analysis of 65 diabetic N2 rats in the TM cross placed the locus in a genomic segment of $3.1 \mathrm{cM}$ between D11M16Mit14 and D11Yokl. The mouse intersubspecies backcross consisting of 1,655 progeny refined the orthologous segment to $3.0 \mathrm{cM}$ between D16Mit14 and D16Mit46, allowing construction of a physical map of the region in both species.

\section{Physical mapping of Iddm/kdpl and genome analysis}

We constructed a physical map using YAC and BAC libraries of rat and mouse [16]. The $I d d m / k d p l$ critical region is covered entirely by rat clones and partially by mouse clones. By sequencing these clones and by searching with BLAST, we identified two known genes, activated leukocyte cell adhesion molecule (Alcam) and $C b l b$, in the critical interval. ALCAM is a CD6 ligand, and CD6-ALCAM interaction may be involved in thymocyte-thymic epithelial cell adhesion and regulation of T-cell function [2], suggesting Alcam as a potential candidate for $I d d m / k d p l$. Sequence analysis of Alcam cDNA showed no obvious mutation except the missense variation Val184Leu, and RT-PCR analysis indicated that mRNA expression was not altered in KDP rats. However, the Val184Leu variation is not specific to KDP, as several rat strains have the same variation [17].

\section{Identification of a nonsense mutation in Cblb gene}

$C b l b$ is another potential candidate for $I d d m / k d p l$, as Cblb deficient mice show several autoimmune phenotypes due to enhanced activation of T-cells [1, 3]. Sequence analysis of Cblb cDNA of KDP rats revealed a homozygous nonsense mutation Arg455X [16]. Cblb was identified as an isoform of proto-oncogene $\mathrm{Cbl}$ (cCbl) [9], and functions as a ubiquitin protein ligase [6] consisting of a tyrosine kinase binding domain, a ring finger domain, several proline rich regions, and a leucine zipper domain. The KDP mutation truncates the protein in the middle, deleting the proline rich regions and leucine zipper domain. The proline rich regions are required for binding of Cblb to its target molecules [6]. Accordingly, the mutation in KDP rats should be loss-of-function, which implies responsibility in the development of the disease in KDP rats.

\section{Functional role of Cblb in $T$ cells}

Signaling through the T-cell receptor and co-stimulatory signaling through $\mathrm{CD} 28$ are both required for T-cell activation (See Fig. 1). In the resting state, Cblb ubiquitinates and inhibits PI3-kinase, an important molecule in CD28 signaling [6]. Upon stimulation, CD28 signaling inhibits Cblb [18], resulting in the activation of PI3-kinase and the T cells. Thus, Cblb is critical in the regulation of T-cell activation. Cblb deficient mice show abnormal T-cell activation in the absence of CD28 signaling, and exhibit several features of autoimmune disease, including lymphocyte infiltration into multiple tissues and production of autoantibody, but do not develop diabetes [1]. Thus, Cblb is an important regulator of autoimmunity, suggesting that dysregulation of the signaling pathway in which Cblb is involved may contribute to human autoimmune diseases including type 1 diabetes. However, disease causing mutations in $C B L B$ have not been reported so far. Association studies of polymorphisms of $C B L B$ in type 1 diabetes found that $C B L B$ is not a major genetic factor in the disease in U.K. and U.S. multiplex type 1 diabetic families [11, 13]. The relationship of $C B L B$ with other autoimmune diseases may be clarified by mutation screenings and association studies.

\section{Histological analysis of the KDP rat}

We also performed histological analysis of the KDP rat [16]. Severe lymphocyte infiltration into the thyroid gland and mild to moderate lymphocyte infiltration into various tissues in addition to the pancreatic islets was observed, including the submandibular gland, kidney, adrenal and pituitary. The histological findings are similar to those in Cblb deficient mice, excepting the severe insulitis in KDP rats.

\section{Transgenic rescue experiment}

To confirm responsibility of the Cblb mutation in the KDP phenotype, we carried out a transgenic rescue experiment [16]. Wild-type Cblb cDNA under regulation of mouse $\mathrm{H}-2 \mathrm{Kd}$ promoter was used as a transgene construct. $\mathrm{H}-2 \mathrm{Kd}$ promoter leads to ubiquitous expression of the transgene. We obtained two independent transgenic lines for the $\mathrm{H}-2 \mathrm{Kd}$ promoter. At 120 days of age, transgenic line 1 was completely rescued from the development of diabetes, and transgenic line 2 was rescued partially but significantly. These data show 


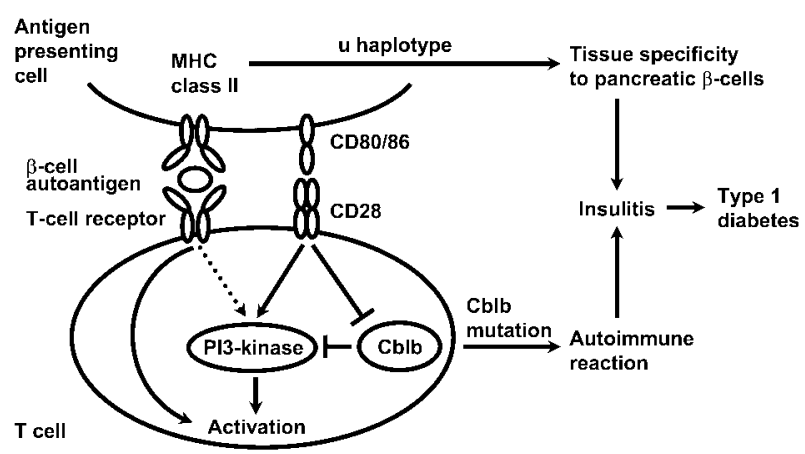

Fig. 1. Two-gene model of the development of type 1 diabetes in the KDP rat. Both Cblb and MHC class II genes are involved in the development of diabetes in this model, the former as a critical regulator of T-cell activation and the latter in antigen presentation to $\mathrm{T}$ cells. The $\mathrm{Cblb}$ mutation leads to autoimmune reaction, and the MHC class II u haplotype confers tissue specificity to the pancreatic $\beta$ cells. In combination, these two states cause insulitis and type 1 diabetes in the KDP rat.

clearly that $\mathrm{Cblb}$ is responsible in the development of diabetes in the KDP rat.

\section{Model of development of type 1 diabetes in the KDP rat}

We suggest a two-gene model of the development of type 1 diabetes in the KDP rat in which two major genes are involved in the development of the disease (Fig. 1). Cblb determines autoimmune reaction while the MHC confers tissue specificity to pancreatic $\beta$-cells. The combination of these two states readily causes insulitis, and results in type 1 diabetes. The Cblb mutation brings about abnormal $\mathrm{T}$-cell activation and lymphocyte infiltration to various tissues, which leads to autoimmune reaction. The MHC class II u haplotype of the KDP rat is frequently distributed in rat strains, and all of the spontaneous and interferon-alpha inducer poly IC-induced models of type 1 diabetes in rats have this haplotype [5]. These data suggest that this class II molecule has specific binding capacity to an unidentified $\beta$-cell autoantigen that activates $\beta$-cell reactive $\mathrm{T}$-cells, thus conferring susceptibility to insulitis and type 1 diabetes. The relationship between MHC class II haplotypes and tissue specificity in autoimmunity may be clarified using congenic strains having specific MHC class II haplotypes and the Cblb mutation.

\section{Conclusion}

We have shown that $C b l b$ is a major susceptibility gene for type 1 diabetes in the KDP rat and that $\mathrm{Cblb}$ functions as a negative regulator of autoimmunity. We propose a two-gene model of the development of type 1 diabetes in which Cblb, in combination with the MHC, accounts for most of the genetic predisposition to the disease. It remains to be determined whether or not $C B L B$ contributes to human autoimmune diseases.

\section{Acknowledgments}

In memory of Professor Dr. K. Komeda (Tokyo Medical University), the developer of the KDP rat, who died unexpectedly in January, 2003. I thank Dr. S. Seino (Kobe University), Dr. T. Serikawa (Kyoto University), and many colleagues for their suggestions and help. This work was supported by Grants-in-Aid for Scientific Research from the Ministry of Education, Culture, Sports, Science and Technology, Japan.

\section{References}

1. Bachmaier, K., Krawczyk, C., Kozieradzki, I., Kong, Y.Y., Sasaki, T., Oliveira-dos-Santos, A., Mariathasan, S., Bouchard, D., Wakeham, A., Itie, A., Le, J., Ohashi, P.S., Sarosi, I., Nishina, H., Lipkowitz, S., and Penninger, J.M. 2000. Negative regulation of lymphocyte activation and autoimmunity by the molecular adaptor Cblb. Nature 403: 211-216.

2. Bowen, M.A., Patel, D.D., Li, X., Modrell, B., Malacko, A.R., Wang, W.C., Marquardt, H., Neubauer, M., Pesando, J.M., and Francke, U. 1995. Cloning, mapping, and characterization of activated leukocyte-cell adhesion molecule (ALCAM), a CD6 ligand. J. Exp. Med. 181: 22132220.

3. Chiang, Y.J., Kole, H.K., Brown, K., Naramura, M., Fukuhara, S., Hu, R.-J., Jang, I.K., Gutkind, J.S., Shevach, E., and Gu, H. 2000. Cblb regulates the CD28 dependence of T-cell activation. Nature 403: 216-220.

4. Davies, J.L., Kawaguchi, Y., Bennett, S.T., Copeman, J.B., Cordell, H.J., Pritchard, L.E., Reed, P.W., Gough, S.C., Jenkins, S.C., Palmer, S.M., Balfour,K.M., Rowe, B.R., Farrall, M., Barnett, A.H., Bain, S.C., and Todd, J.A. 1994. A genome-wide search for human type 1 diabetes susceptibility genes. Nature 371: 130-136.

5. Ellerman, K.E. and Like, A.A. 2000. Susceptibility to diabetes is widely distributed in normal class II" haplotype rats. Diabetologia 43: 890-898.

6. Fang, D., Wang, H.Y., Fang, N., Altman, Y., Elly, C., and Liu, Y.C. 2001. Cbl-b, a RING-type E3 ubiquitin ligase, 
targets phosphatidylinositol 3-kinase for ubiquitination in T cells. J. Biol. Chem. 276: 4872-4878.

7. Hornum, L., Romer, J., and Markholst, H. 2002. The diabetes-prone BB rat carries a frameshift mutation in Ian4, a positional candidate of Iddm1. Diabetes 51: 1972-1979.

8. Kawano, K., Hirashima,T., Mori, S., Saitoh, Y., Kurosumi, M., and Natori, T. 1991. New inbred strain of Long-Evans Tokushima Lean rats with IDDM without lymphopenia. Diabetes 40: 1375-1381.

9. Keane, M.M., Rivero-Lezcano, O.M., Mitchell, J.A., Robbins, K.C., and Lipkowitz, S. 1995. Cloning and characterization of Cblb: a SH3 binding protein with homology to the c-cbl proto-oncogene. Oncogene 10: 23672377.

10. Komeda. K., Noda, M., Terao, K., Kuzuya, N., Kanazawa, M., and Kanazawa, Y. 1998. Establishment of two substrains, diabetes-prone and non-diabetic, from LongEvans Tokushima Lean (LETL) rats. Endocr. J. 45: 737-744.

11. Kosoy, R., Yokoi, N., Seino, S., and Concannon, P. 2004. Polymorphic variation in the $C B L B$ gene in human type 1 diabetes. Genes Immun. 5: 232-235.

12. MacMurray, A.J., Moralejo, D.H., Kwitek, A.E., Rutledge, E.A., Van Yserloo, B., Gohlke, P., Speros, S.J., Snyder, B., Schaefer, J., Bieg, S., Jiang, J., Ettinger, R.A., Fuller, J., Daniels, T.L., Pettersson, A., Orlebeke, K., Birren, B., Jacob, H.J., Lander, E.S., and Lernmark, A. 2002. Lymphopenia in the BB rat model of type 1 diabetes is due to a mutation in a novel immune-associated nucleotide
(Ian)-related gene. Genome Res. 12: 1029-1039.

13. Payne, F., Smyth, D.J., Pask, R., Barratt, B.J., Cooper, J.D., Twells, R.C., Walker, N.M., Lam, A.C., Smink, L.J., Nutland, S., Rance, H.E., and Todd, J.A. 2004. Haplotype tag single nucleotide polymorphism analysis of the human orthologues of the rat type 1 diabetes genes Ian4 (Lyp/ $I d d m 1$ ) and Cblb. Diabetes 53: 505-509.

14. Wicker, L.S., Todd, J.A., and Peterson, L.B. 1995. Genetic control of autoimmune diabetes in the NOD mouse. Аnпи. Rev. Immunol. 13: 179-200.

15. Yokoi, N., Kanazawa, M., Kitada, K., Tanaka, A., Kanazawa, Y., Suda, S., Ito, H., Serikawa, T., and Komeda, K. 1997. A non-MHC locus essential for autoimmune type I diabetes in the Komeda diabetes-prone rat. J. Clin. Invest. 100: 2015-2021.

16. Yokoi, N., Komeda, K., Wang, H.-Y., Yano, H., Kitada, K., Saitoh, Y., Seino, Y., Yasuda, K., Serikawa, T., and Seino, S. 2002. Cblb is a major susceptibility gene for rat type 1 diabetes mellitus. Nature Genet. 31: 391-394.

17. Yokoi, N., Namae, M., Fuse, M., Wang, H.-Y., Hirata, T., Seino, S., and Komeda, K. 2003. Establishment and characterization of the Komeda diabetes-prone rat as a segregating inbred strain. Exp. Anim. 52: 295-301.

18. Zhang, J., Bardos, T., Li, D., Gal, I., Vermes, C., Xu, J., Mikecz, K., Finnegan, A., Lipkowitz, S., and Glant, T.T. 2002. Cutting edge: regulation of $\mathrm{T}$ cell activation threshold by $\mathrm{CD} 28$ costimulation through targeting Cbl-b for ubiquitination. J. Immunol. 169: 2236-2240. 